\title{
A New Modulation Index Control (MIC) Technique for Reducing THD for Photovoltaic Applications
}

\author{
Asmaa El-Hussiny \\ National Organization for Potable \\ Water and Sanitary Drainage \\ Egypt \\ AsmaaaElhosainy@gmail.com
}

\author{
Hany A. Hamed \\ Emirates Steel. Abu Dhabi, United \\ Arab Emirates \\ Hanyhamed.phd@gmail.com
}

\author{
E. E. El-Kholy \\ Electrical Dept., Faculty of \\ Engineering, Menoufia University \\ Egypt \\ e.e.el-kholy@ieee.org
}

\begin{abstract}
This paper proposes a new technique to enhance the inverter output wave quality by introducing a new technique which optimizes the inverter modulation index and it is so-called Modulation Index Controller (MIC). Therefore, the MIC technique ensures optimum dc-link voltage which results in modulating the inverter output Total Harmonic Distortion (THD). In addition, extracting the maximum PV power by controlling directly the PV load without controlling the duty cycle of the conventional DC-DC converters. This paper presents a three-level Neutral-Point-Clamped (3L-NPC) multilevel inverter interfaced Photovoltaic energy conversion system to generate output voltage with low total harmonic distortion via Buck-boost converter which regulates the DC-Link voltage by increasing the modulation index to reduce Total Harmonic Distortion (THD). The system is modeled using MATLAB/SIMULINK. Moreover, the system has been experienced when using MIC technique and without using MIC technique. The simulation and experimental results proved that the overall proposed system including Direct Power MPPT and MIC control decline the total harmonic distortion and enhancing the overall system efficiency.
\end{abstract}

Keywords-P\&O, Photovoltaic, DC-link Voltage, NPC-MLI, Closed loop Power Control, THD, Modulation Index Controller (MIC)

\section{INTRODUCTION}

Due to the penetration of solar systems in grid or a standalone generation system to supply different loads, it is necessary to interface the solar system with a DC/AC converter to get $\mathrm{AC}$ voltage. Multilevel inverters are the good choice compared to conventional two-level inverters due to their features such as reducing the total harmonic distortion (THD) and minimizing voltage stress on the semiconductor devices. Also, it is necessary to use a DC/DC converter such as a buck-boost converter between a solar array and multilevel inverters to regulate the dc output voltage of PV. Changing in atmospheric conditions and partial shading conditions PSC are the most popular drawbacks of PV systems which result in lower power output and lower efficiency. Therefore, improving control techniques to enhance the performance of $\mathrm{PV}$ is essential to achieve maximum power under those conditions [1]. Other studies have focused on developing the conventional voltage source inverter for the multilevel implementation [2]. On the other hand, the research has been extended to investigate the performance of various modulation techniques to enhance the performance of MLIs and as well optimize its operation under different configurations. The adopted modulation techniques in multilevel inverters are commonly classified according to their switching frequency range. There is a limitation to use high-frequency modulations techniques in medium voltage range as the switching losses will degrade the inverter rating and reduce its overall efficiency. The typical level or phase shifted carrier-based sinusoidal PWM (SPWM) modulation technique is commonly adopted in industrial applications as the harmonic content on the produced voltage wave is minimized. Another commonly used modulation technique is the so-called Space Vector Modulation (SVM) which has a distinguished performance in three-level topologies. The low switching frequency modulation techniques such as staircase modulation, Space-Vector Control (SVC) and selective harmonic elimination have gained more popularity and interest for a broad range of applications [3].

Modulation index has a crucial in all the techniques. Modulation can be overmodulation or under modulation depending on modulation ratio, and accordingly, total harmonic distortion (THD) varies. Multilevel inverters can be controlled using different modulation techniques. These techniques can be classified according to the switching frequency as follows [4]: Fundamental switching frequency modulation (FSFM) and High switching frequency modulation (HSFM). For both cases, the staired output waveform is obtained, but when using the high switching frequency methods, steppes are modulated with some PWM.

The proposed system is tested for a standalone PV system fed inductive resistive load, based on Matlab/Simulink, including PV array, maximum power point tracking MPPT technique, DC-DC Buck-Boost converter and three-level NPC multilevel inverter. In this paper, novel three different control methods are proposed and analyzed. PMPPT technique based direct load power control aims to increase the overall efficiency by extracting the maximum power of the PV module. Based on direct load power control, this paper will focus on the perturb and observe method, which controls the PV terminal voltage indirectly. Direct load power control will be introduced to show how to obtain the maximum power of the PV module. This new algorithm is called Power Maximum Power Point Tracking (PMPPT)

technique. Eventually, The proposed algorithm increases the overall efficiency. In addition to this, This paper introduces the ability of buck-boost converter to regulate the dc-link voltage. DC-Link voltage control aims to decrease THD by increasing modulation index. Modulation index contren is generating switching pulses of NPC inverter. Thuts, the objective of the proposed DC-Link control is 
modulation index to generate ac output voltage with low Total Harmonic Distortion (THD). Experimental results show a well matching with the simulation ones and approved the highly improved system performances such as the robustness of the system and the system efficiency.

This paper has been organized as follows, Section 1 presents an introduction of different types of control techniques of MLIs, followed by an overview of the neutral-point-clamped has been presented in Section 2. Proposed control techniques are discussed in section 3. Simulation results have been elaborated in Section 4. Section 5 elaborated the experimental setup of the proposed system. The experimental results are introduced in section 6. Finally, section 8 represents the conclusion.

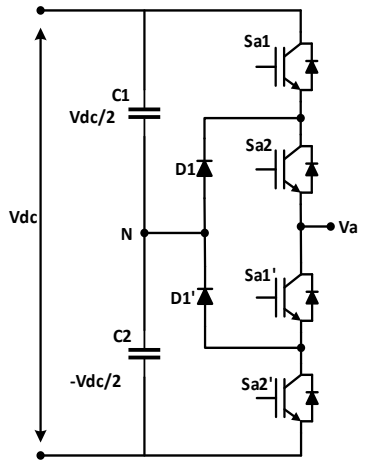

Fig. 1. Circuit configuration of One phase leg for 3L-NPC inverter.

\section{NEUTRAL-POINT-CLAMPED MULTI-LEVEL INVERTER OVERVIEW}

The NPC inverter is the most widely used in all types of industrial applications [5], [6], in the range of 2.3 to $4.16 \mathrm{kV}$, with some applications up to $6 \mathrm{kV}$ [7]. A NPC inverter is basically composed of two traditional two-level VSC stacked one over the other with some minor modifications. As can be seen in Fig. 1,2. the negative bar of the upper converter and the positive bar of the lower are joined together to form the new phase output, while the original phase outputs are connected via two clamping diodes to form the neutral point $\mathrm{N}$, dividing the dc-link voltage in two. Now each power device has to block only the half of the total converter voltage, hence with the same semiconductor [8].

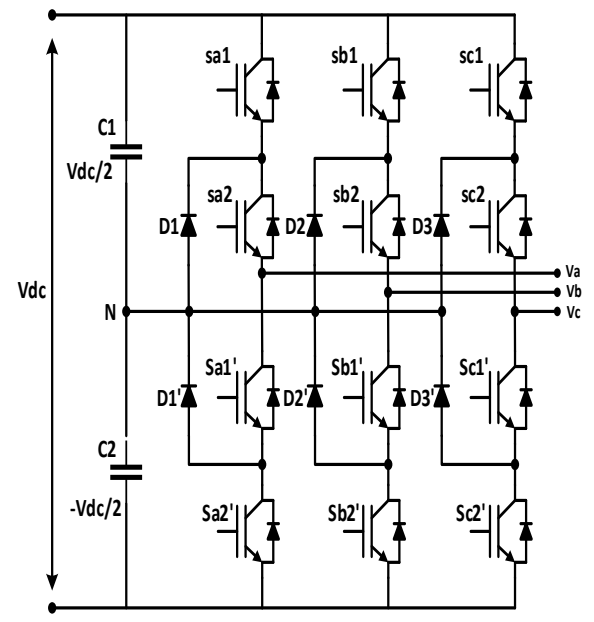

Fig. 2. Circuit configuration of three-phase leg for 3L-NPC inverter.
The NPC inverters are efficient in fundamental frequency switching range hence they are used efficiently in high power medium voltage grid connected converters. The benefit of this topology is its ability to reduce the harmonic contents in the produced voltage by adding the zero-voltage level. Higher levels can be obtained by increasing the switching devices, clamping diodes and capacitors [9], [10], [11], [12]. But the disadvantages include relatively high switching losses which limit the switching frequency to a couple hundred hertz [13], unsymmetrical semiconductor loss distribution, the necessity of a sine filter for standard machines [14], and the difficult extension of the voltage converter range for use by semiconductors with a higher blocking capability or a series connection of semiconductors [15].

\section{PROPOSED CONTROL TECHNIQUES}

Fig 3. Shows the block diagram of the overall proposed system. The proposed system consists of three control techniques; closed loop power control which achieving MPPT by directly controlling load power, Modulation Index Control Loop (MIC) which controlling the inverter modulation index and finally voltage control loop which regulating dc-link voltage. Each part of the control techniques is clearly discussed in the upcoming sections.

The per unit power reference is obtained through a maximum power point tracking (PMPPT) technique (P\&O algorithm) and compared to the actual value of load power. The resulting error is processed through a PI controller with the appropriate gains to obtain the value of actual modulation index and generate the required pulses to the inverter through a conventional PWM modulator. The actual modulation index value is compared to the corresponding command value $\left(\mathrm{m}^{*}\right)$. The resulting error is processed through a PI controller to obtain $(\Delta \mathrm{Vdc})$, which is subtracted from the value of the dclink voltage ( 100 volt) to obtain the final dc-link voltage value. The sensed dc-link voltage value is compared to the value of dc-link voltage reference (Vdc ref). The resulting error is processed through a PI controller with the appropriate gains and a saturation block to obtain the duty ratio of the buckboost converter and generate the required pulse to the BuckBoost converter by PWM modulator.

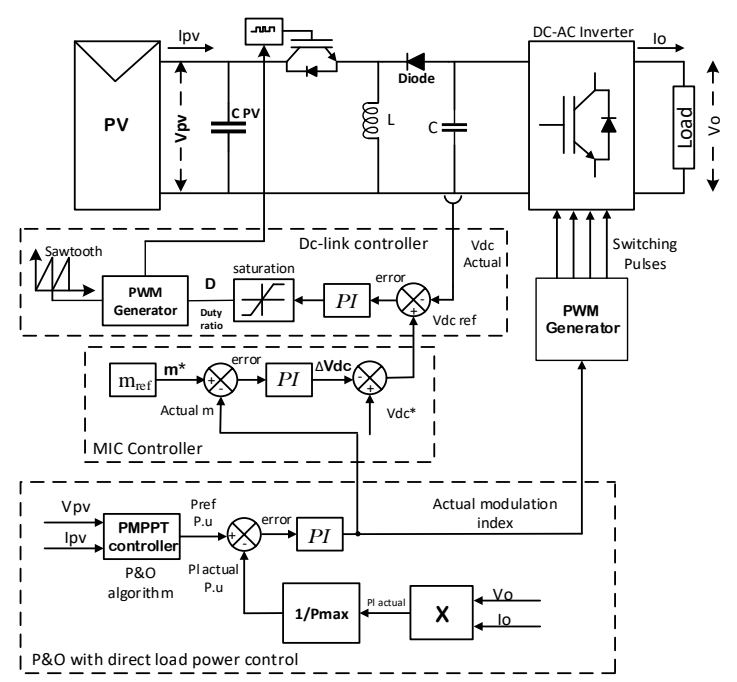

Fig. 3. Block Diagram of the proposed system. 
International Journal of Applied Energy Systems, Vol. 2, No. 2, July 2020 ISSN: 2636 - 3712 (Printed Version) ISSN: 2636 - 3720 (Online Version) Special Issue: ICEE-2019

\section{A. Block diagram of the closed loop power control}

The voltage and current generated by photovoltaic array are inputs of the PMPPT Controller as shown in Fig. 4. and the task of the PMPPT Controller is to calculate the per unit power reference. The PMPPT Technique contains two control loops to achieve maximum power. The inner control loop includes the PMPPT Inverter Controller (P\&O algorithm) block and a PI controller to generate the modulation index then generates the modulating signal, comparing this modulating signal with two symmetrical in-phase level shifted triangle carries, PWM signals are generated (the switching pulses of the multilevel inverter). The external control loop is to calculate the per unit power reference (contains the direct load power control). The PMPPT block works towards minimizing the error between power reference (Pref) and the load power (Pactual). The PI controller parameters were adjusted after many trails until the values shown in TABLE I.

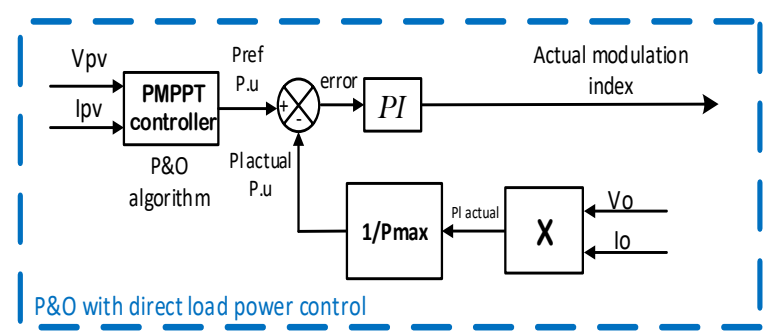

Fig. 4. Block Diagram of the closed loop power control.

TABLE I. PARAMETERS OF THE PI CONTROLLER FOR POWER CONTROL LOOP.

\begin{tabular}{ccc}
\hline Gain & Kp & Ki \\
\hline Value & 1.5 & 20 \\
\hline
\end{tabular}

\section{B. Block Diagram of the Modulation Index Control Loop} (MIC)

The modulation index reference $\left(\mathrm{m}^{*}=0.95\right)$ is compared to the actual modulation index and of the error is passed to the MIC Controller as shown in Fig. 5. The MIC controller generates the dc-link voltage reference ( $\mathrm{Vdc}$ ref). The reference modulation index (for MIC controller is considered as the feedback actual value) is generated from PMPPT controller. The PI controller parameters were adjusted after many trails until the values shown in TABLE II.

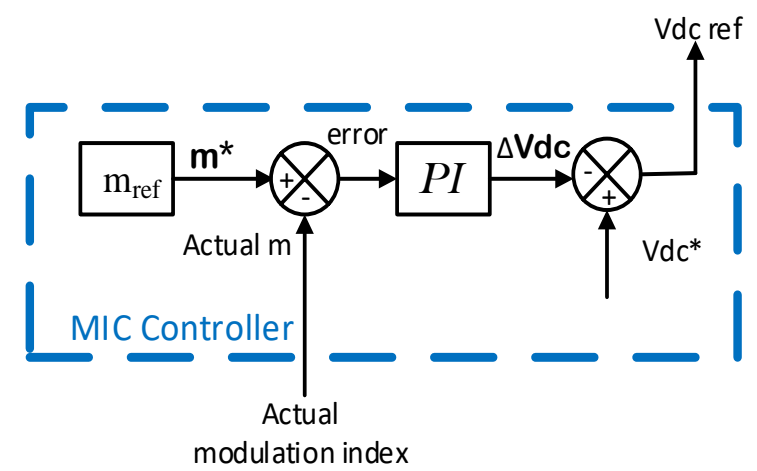

Fig. 5. Block Diagram of the modulation index control.
TABLE II. PARAMETERS OF THE PI CONTROLLER FOR MIC CONTROL LOOP.

\begin{tabular}{ccc}
\hline Gain & Kp & Ki \\
\hline Value & 10 & 500 \\
\hline
\end{tabular}

\section{Block Diagram of Voltage Control Loop}

As shown in Fig. 6. The dc-link voltage reference and actual dc -link voltage (output voltage of DC-DC Buck-Boost converter) are inputs of the DC-Link controller. The task of the DC-Link controller is to generate the duty ratio of the buck-boost converter (the switching pulses). Comparing this duty ratio to a sawtooth, a PWM is generated. The saturation block is to limit the duty ratio between 0.1 and 0.8 . The PI controller parameters were adjusted after many trails until the values shown in TABLE III.

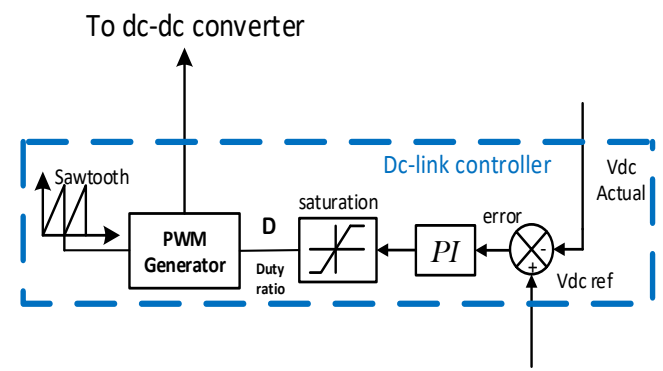

Fig. 6. Block Diagram of the closed loop voltage control.

TABLE III. TABLE rPARAMETERS OF THE PI CONTROLLER FOR VOLTAGE CONTROL LOOP.

\begin{tabular}{ccc}
\hline Gain & Kp & Ki \\
\hline Value & 0.2 & 5 \\
\hline
\end{tabular}

\section{Simulation RESUltS}

Simulation is performed by MATLAB/Simulink software package. In order to validate the proposed MIC control technique. Simulation results are introduced for each part; without implementing the MIC control technique, and with MIC technique. The system used in the simulation consists of a DC-DC buck-boost converter, NPC multilevel inverter, and $\mathrm{RL}$ load $(\mathrm{R}=5 \Omega$ and $\mathrm{L}=3 \mathrm{mH})$. Firstly, the irradiation is set to $1000 \mathrm{~W} / \mathrm{m} 2$ and the panels temperature is set to 25 degrees. At the start of simulation, the PV is initialized at time equals to 0 seconds, then when the PV output voltage is stable at no load condition, the inverter is enabled with initial load based on a fixed modulation index at time equals to 0.1 seconds. The dc-link controller is enabled to fix the dc voltage to the desired level at time equals 0.1 seconds. Once the dc-link is stable, the MPPT algorithm is enabled at time equals 1 seconds to extract the maximum power from PV, and the MPPT generates the power reference to the inverter. When the system is stable, the MIC controller is enabled at time equals to 3 seconds to enhance the performance of the PV system by regulating the dc-link voltage which resulted in lowering the overall harmonics.

\section{A. Testing the proposed MIC technique}

Using MATLAB/SIMULINK simulation program, the system was tested for different values of modulation index (m). 
TABLE IV. summarizes the results that have been recorded for different values of modulation index. It can be noted that, the best three values of modulation index which result in low total harmonic distortion at $m=0.95,0.96$, and 0.97 . the value of the reference modulation index selected in this thesis is 0.95 .

TABLE IV. VARIATION OF THD \% VERSUS MODULATION INDEX USING MIC TECHNIQUE.

\begin{tabular}{|c|c|}
\hline $\mathbf{m}$ & $\begin{array}{c}\text { Total Harmonic } \\
\text { Distortion (THD\%) }\end{array}$ \\
\hline 0.8 & 8.25 \\
\hline 0.85 & 7.35 \\
\hline 0.9 & 6.33 \\
\hline 0.91 & 6.92 \\
\hline 0.92 & 6.37 \\
\hline 0.93 & 6.55 \\
\hline 0.94 & 6.40 \\
\hline 0.95 & 6.27 \\
\hline 0.96 & 6.06 \\
\hline 0.97 & 6.20 \\
\hline 0.98 & 6.63 \\
\hline 0.99 & 6.36 \\
\hline 1.0 & 6.64 \\
\hline
\end{tabular}

\section{B. Performance Test}

1) System without using MIC technique

It can be noted that when the MIC is off, the dc-link voltage is constant at 100 volt all over the period, and the modulation index equals 0.82 . Simulation results are illustrated at Fig. 7.
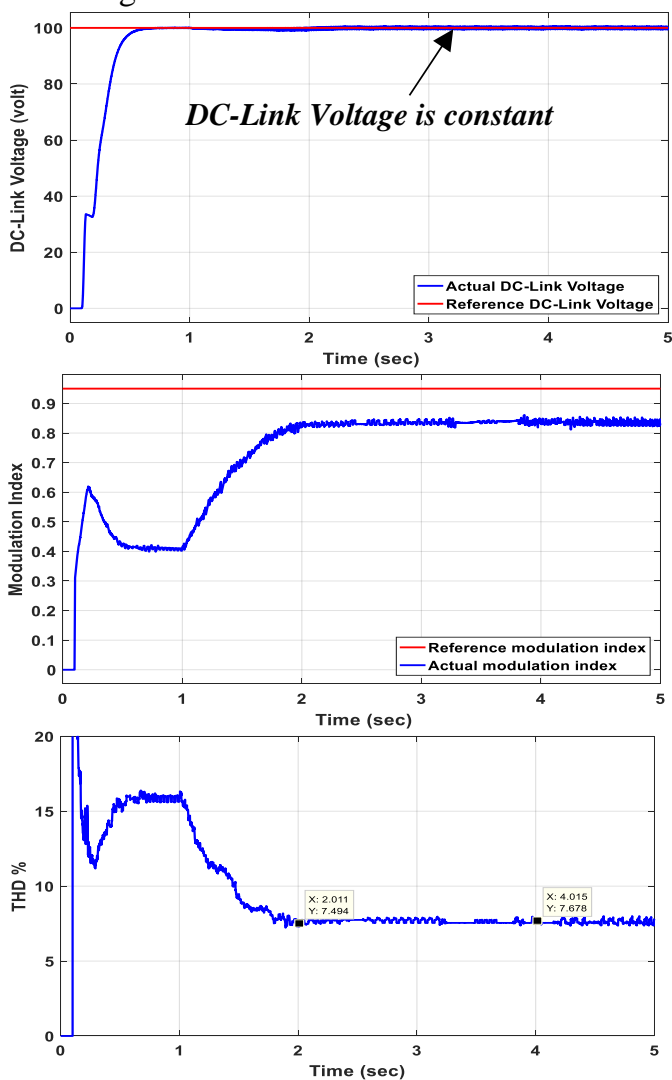

Fig. 7. Simulation Results for dc-link voltage, modulation indxe and THD without MIC technique under steady weather conditions $(1000 \mathrm{~W} / \mathrm{m} 2$ at 25 $\mathrm{oC})$.

\section{2) System using MIC technique}

In this case, the MIC control is on at time equals 3 second. It can noticed that the inverter voltage is regulated after enabled MIC control and it dropped from 100 volt to 87 volt at this moment the modulation index soared from 0.82 to 0.94 . Thus, the total harmonic distortion minimized from $7.6 \%$ to $6.24 \%$ at the end of the period. Simulation results are illustrated at Fig. 8. The proposed MIC control exhibits good performance in enhancing the inverter output by regulating the dc-link voltage which resulted in lowering the overall harmonics as shown in Fig. 9,10.
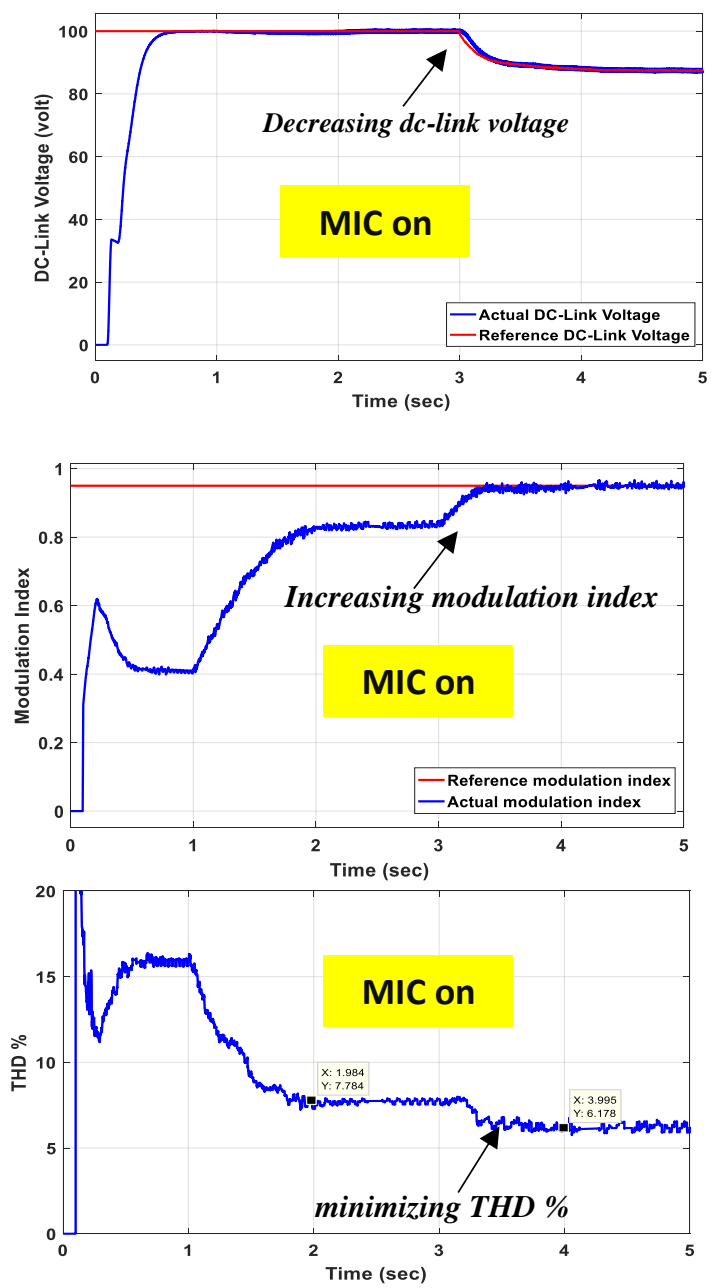

Fig. 8. Simulation Results for dc-link voltage, modulation index and THD with MIC technique under steady weather conditions $(1000 \mathrm{~W} / \mathrm{m} 2$ at 25 $\mathrm{oC})$.

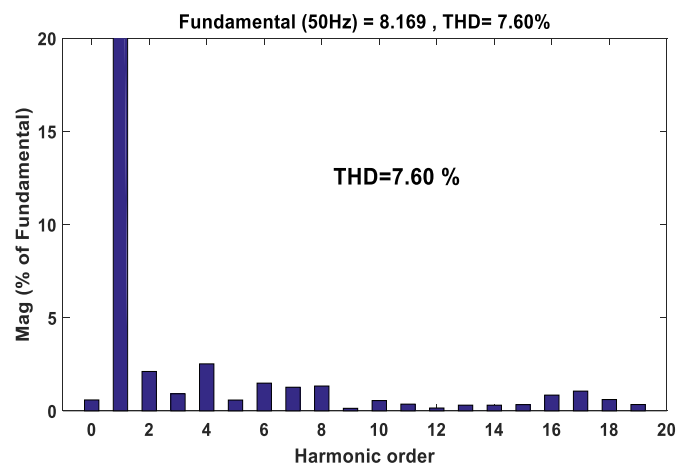

Fig. 9. Simulated spectrum analysis for inverter load current without MIC technique. 
International Journal of Applied Energy Systems, Vol. 2, No. 2, July 2020 ISSN: 2636 - 3712 (Printed Version) ISSN: 2636 - 3720 (Online Version) Special Issue: ICEE-2019

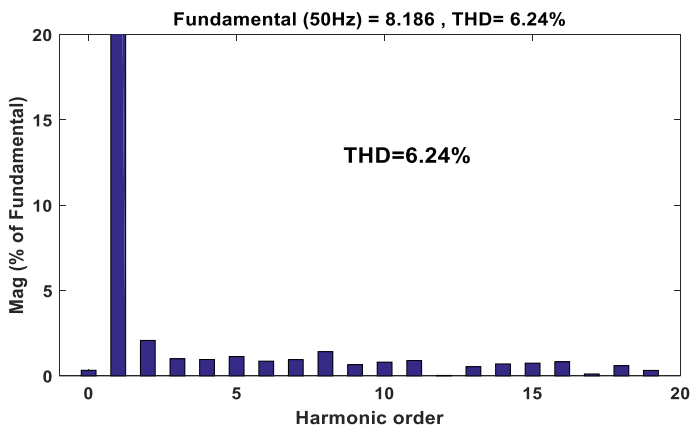

Fig. 10. Simulated spectrum analysis for inverter load current with MIC technique.

\section{EXPERIMENTAL SETUP}

An experimental setup was designed to evaluate the proposed technique. The hardware was directly connected to Simulink using dSPACE DS1104 embedded card. A buckboost converter similar to simulation setup was used for practical confirmation. The output of proposed MPPT P\&O algorithm and MIC control technique is fed to the DS1104 block, which generates the switching PWM signal at desired frequency. PWM signal is then fed to gate driver that gates the power IGBT.

\section{EXPERIMENTAL RESULTS}

This experimentation was obtained at irradiation $=980 \mathrm{w} / \mathrm{m} 2$ and Temperature $=30{ }^{\circ} \mathrm{C}$ at the mid-day during July of 2018 . Fig. 11. shows the experimental results of the MPPT and proposed MIC control technique. It is observed that the output power almost equals the input PV power. This means less power loss. Therefore, the efficiency of the proposed system is very high and extremally equals $98 \%$. Also, the inverter load current has a low THD and equals circa $6.24 \%$.

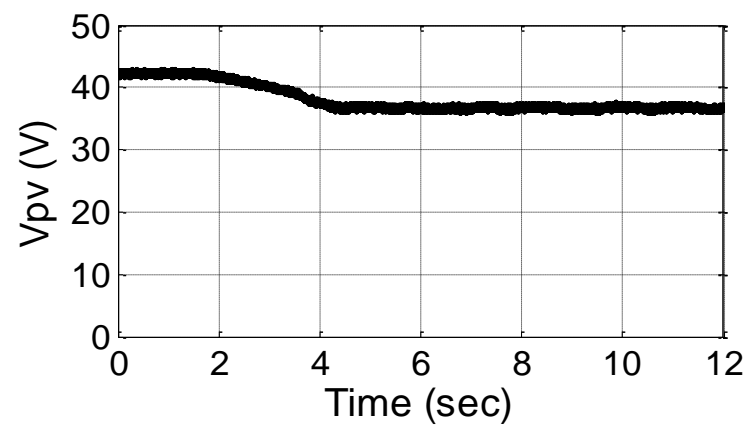

(a)

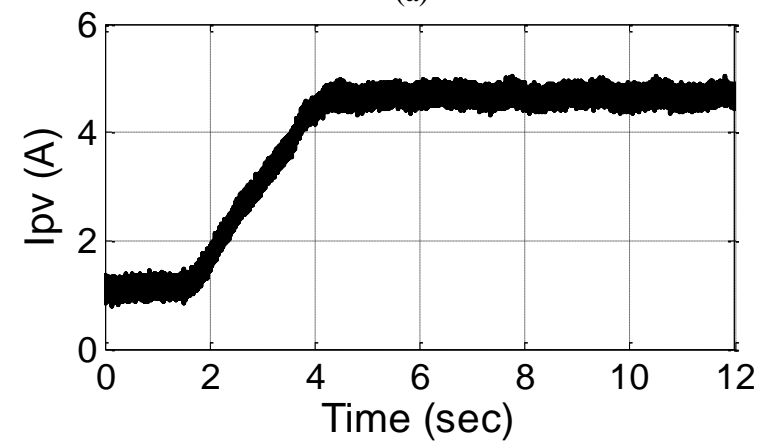

(b)

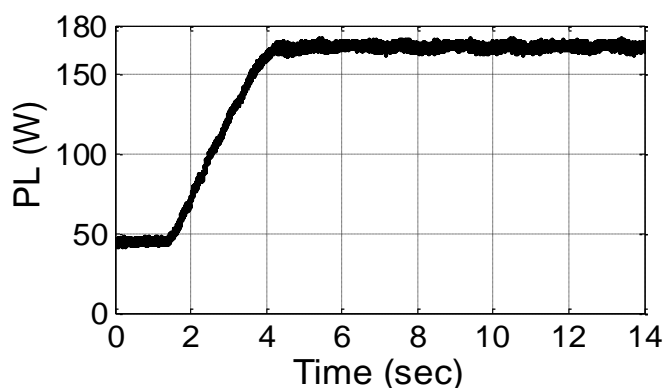

(c)

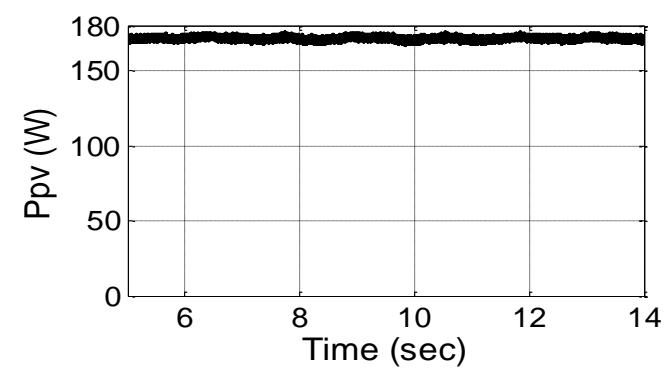

(d)

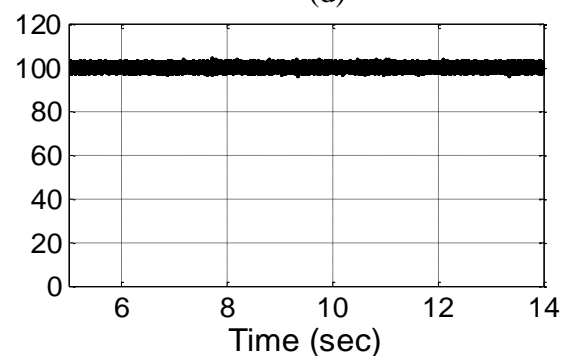

(e)

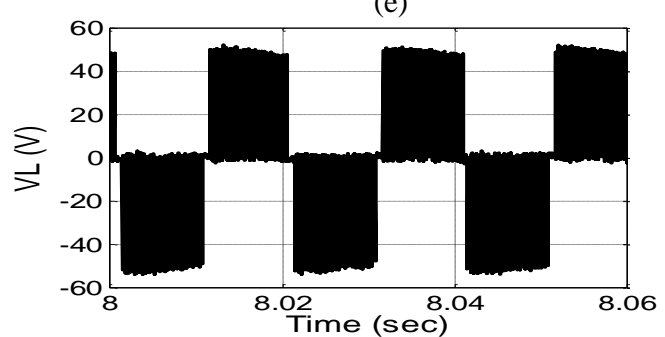

(f)

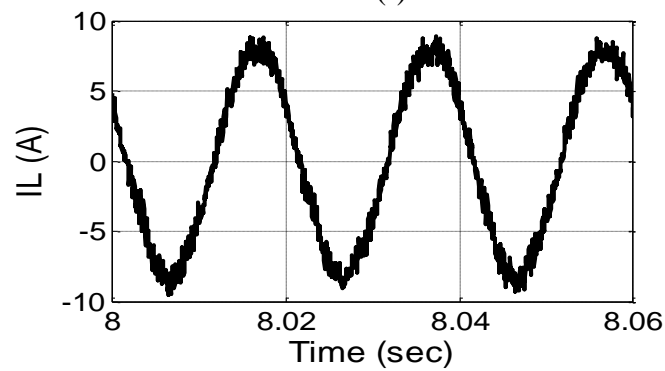

(g)

Fig. 11. Experimental results (a) PV Voltage, (b) PV Current, (c) Load Power, (d) PV Power, (e) Dc-Link Voltage. (f) Load Voltage, and (g) Load Current.

\section{CONCLUSIONS}

In this paper, a three-level Neutral-Point-Clamped (3LNPC) multilevel inverter interfaced Photovoltaic energy conversion system has been presented. The salient feature of the model is that it has a new control technique for enhancing inverter output wave quality that allows to regulate the inverter dc link voltage to minimize total harmonic distortion 
of the inverter output. The MIC technique ensures optimum dc-link voltage which results in enhancing the inverter output Total Harmonic Distortion (THD). The system was firstly testing without using MIC technique and then was testing with applying MIC controller. The results indicate that reduced THD from $7.6 \%$ to $6.24 \%$ by applying MIC control technique. Also, The efficiency of the overall system is increased by applying the proposed control techniques. The results have been checked using a designed prototype integrated into a full laboratory setup.

\section{Appendix A}

PV modul parameters.

\begin{tabular}{ll}
\hline Maximum Power & $179.928 \mathrm{~W}$ \\
Voltage at MPP & $36.72 \mathrm{~V}$ \\
Current at MPP & $4.9 \mathrm{~A}$ \\
Short-circuit current & $5.31 \mathrm{~A}$ \\
Open-circuit voltage & $44.06 \mathrm{~V}$ \\
\hline
\end{tabular}

\section{Appendix B}

Simulation Parameters.

\begin{tabular}{lc}
\hline Components & Values \\
Input Capacitor & $1000 \mu \mathrm{F}$ \\
Output Capacitor & $1000 \mu \mathrm{F}$ \\
Inductor & $10 \mathrm{mH}$ \\
Caocitor of DC-Link & $9000 \mu \mathrm{F}$ \\
Load & $5 \mathrm{ohm}$ and $3 \mathrm{mH}$ \\
Switching Frequency & $25 \mathrm{kHz}$ \\
\hline
\end{tabular}

REFERENCES

[1] M. A. M. Ramli, S. Twaha, K. Ishaque, and Y. AI-Turki, "A Review on Maximum Power Point Tracking for Photovoltaic Systems with and Without Shading Conditions," Renewable and Sustainable Energy Reviews, vol. 67, pp. 144-159, 2017.

[2] F. Khoucha, M. S. Lagoun, A. Kheloui, and M. E. H. Benbouzid, "A Comparison of Symmetrical and Asymmetrical Three-Phase H-Bridge Multilevel Inverter for DTC Induction Motor Drives," IEEE Transactions on Energy Conversion, vol. 26, no. 1, pp. 64-72, 2011.

[3] J. Rodriguez, L. Jih-Sheng, and P. Fang Zheng, "Multilevel inverters: a survey of topologies, controls, and applications," IEEE Transactions on Industrial Electronics, vol. 49, no. 4, pp. 724-738, 2002.

[4] J. Rodriguez, L. Moran, P. Correa, and C. Silva, "A vector control technique for medium-voltage multilevel inverters," IEEE Transactions on Industrial Electronics, vol. 49, no. 4, pp. 882-888, 2002.

[5] A. Nabae, I. Takahashi, and H. Akagi, "A New NeutralPoint-Clamped PWM Inverter," IEEE Transactions on Industry Applications, vol. IA-17, no. 5, pp. 518-523, 1981.

[6] R. H. Baker, "Bridge converter circuit," U.S. Patent 4 270 163, May 26, 1981.

[7] P. K. S. j. Rodriguez, I.E. Lizama, “A Survey on NeutralPoint-Clamped Inverters," IEEE Trans. Ind. Electron, vol. 57, no. 7, pp. 2219-2230, July. 2010.
[8] e. a. Rodriguez, "Multilevel converters: An enabling technology for high-power applications," in Proc. the IEEE, vol. vol. 97, pp. 1786-1817., 11, November 2009.

[9] I. Çolak, and E. K. E., "A review on inverter topologies and developments," In: Proceedings of Eleco'2008 electrics, electronics and computer engineering symposium, Bursa (Turkey), 2008.

[10] L. M. Tolbert, F. Z. Peng, and T. G. Habetler, "Multilevel inverters for electric vehicle applications." pp. 79-84. 1998.

[11] A. Bendre, S. Krstic, J. V. Meer, and G. Venkataramanan, "Comparative evaluation of modulation algorithms for neutral-point-clamped converters," IEEE Transactions on Industry Applications, vol. 41, no. 2, pp. 634-643, 2005.

[12] P. P, and S. S., "A new generalized space vector modulation algorithm for neutral-point-clamped multilevel converters," In: Proceedings of progress in electromagnetics research symp, Cambridge (USA), 2006.

[13] M. E. R. D.P.Hohm "Comparative Study of Maximum Power Point Tracking Algorithms Using an Experimental, Programmable, Maximum Power Point Tracking Test Bed," IEEE Proc. of photovoltaic specialists conference, pp. 1699-1702, 2000.

[14] S. Bernet, " Recent developments of high power converters for industry and traction applications," IEEE Trans. Power Electronics, vol. 15, pp. 1102-1117, Nov. 2000.

[15] O. A. P. K. Steimer, B. Ødegård, S. Bernet, and T. Brückner, "Very high power IGCT PEBB technology," in Proc. IEEE Power Electron. Spec. Conf., Recife, Brazil, 2005, pp. 1-7. 